\title{
Positron emission tomography/computed tomography-guided percutaneous trans- pararectal space prostate biopsy for the diagnosis of prostate cancer: A retrospective study
}

\section{Nana Luo}

Xiamen University and Fujian Medical University Affiliated First Hospital

\section{Dan Ruan}

Xiamen University and Fujian Medical University Affiliated First Hospital

Yi-zhen Pang

Xiamen University and Fujian Medical University Affiliated First Hospital

Qi-hang Shang

Xiamen University and Fujian Medical University Affiliated First Hospital Hao-jun Chen

Xiamen University and Fujian Medical University Affiliated First Hospital

Wei Guo

Xiamen University and Fujian Medical University Affiliated First Hospital

\section{Bing Hao}

Xiamen University and Fujian Medical University Affiliated First Hospital

\section{Hua Wu}

Xiamen University and Fujian Medical University Affiliated First Hospital Long Sun (D13178352662@163.com )

\section{Research article}

Keywords: positron emission tomography/computed tomography; prostate cancer; percutaneous transpararectal space prostate biopsy

Posted Date: January 16th, 2020

DOI: https://doi.org/10.21203/rs.2.17523/v3

License: (c) (1) This work is licensed under a Creative Commons Attribution 4.0 International License. Read Full License 


\section{Abstract}

Background Biopsy is considered the gold-standard technique for prostate cancer diagnosis and is recommended in patients with a high clinical indication of prostate cancer. In this study, we aimed to determine the diagnostic efficacy of a novel positron emission tomography (PET)/computed tomography (CT)-guided percutaneous trans-pararectal space-based approach to targeted prostate biopsy.Methods PET/CT-guided percutaneous trans-pararectal space prostate biopsies were performed in 14 consecutive patients with indications of prostate cancer. Whole-body 18 F-FDG PET/CT indicated the presence of 18 F-fluorodeoxyglucose (FDG)-avid focal prostate lesions. Two tissue specimens were obtained from each patient. The final diagnoses were established based on the results of a histopathological analysis and clinical follow-up, and these findings were used to verify the diagnostic accuracy of 18 F-FDG PET/CT for prostate cancer.Results The diagnostic accuracy of 18 F-FDG PET/CT for prostate cancer was $81.8 \%$. Further analyses of the two biopsied samples per patient led to confirmed histopathological and immunohistochemical diagnoses of prostate cancer in all 14 patients. Consequently, the success rate of PET/CT-guided percutaneous trans-pararectal space prostate-targeted biopsy for the diagnosis of prostate cancer was $100.0 \%$ (14/14). Regarding safety, the average duration of biopsy was 20 min, and no serious complications occurred.Conclusions PET/CT-guided percutaneous trans-pararectal space prostate biopsy may yield a new approach to targeted prostate biopsy for the diagnosis of prostate cancer. Moreover, this biopsy procedure can be performed safely without complications, and is more costeffective than conventional trans-rectal and trans-perineal prostate biopsy methods.

\section{Background}

Prostate cancer is an important cause of morbidity and mortality in men worldwide [1]. In China, the incidence of prostate cancer has been increasing by approximately $12 \%$ annually. However, approximately $70 \%$ of patients present with locally advanced or extensively metastasised prostate cancer at the time of diagnosis [2-3]. Historically, trans-rectal ultrasonography (TRUS) of the prostate has remained the cornerstone of prostate cancer diagnosis since the period of systematic 'sextant' biopsy of suspected prostate malignancy, wherein 3 cores are obtained from each side of the prostate [4-6]. In men undergoing an initial prostate needle biopsy consequent to an elevated serum prostate-specific antigen (PSA) level, systematic 12-core TRUS-guided prostate biopsy yields cancer detection rates of 28-52\% [713]. The systematic sampling of all prostatic quadrants improves the accuracy of sampling. However, this method has been criticised for its oversampling of insignificant tumours, the risk of additional morbidity and the need for general anaesthesia.

Compared with traditional biopsy protocols such as systematic 12-core TRUS-guided biopsy, multiparametric magnetic resonance imaging (MRI)/ultrasonography-guided biopsy yields more refined diagnostic information [14-16]. The minor complications of the trans-rectal and trans-perineal biopsy procedure include haematuria, rectal haemorrhage, haemospermia, vasovagal attack, and pathogenic infection. TRUS-guided prostate biopsy is associated with additional major complications, including 
sepsis, bleeding or other complications requiring hospitalisation. More importantly, an increased risk of life-threatening septic shock was reported with trans-rectal biopsy [17].

Therefore, a safer and more effective algorithm for the diagnosis of prostatic diseases should be explored. Recent advancements in molecular imaging techniques (positron emission tomography [PET]/computed tomography [CT], PET/MRI) have led to the development of several new high-specificity probes for the diagnosis of prostate cancer and may play a crucial role in facilitating the management of prostate cancer at all clinical stages [18-20]. In our previous study, we proved the efficacy of PET/CTguided targeted biopsy for the evaluation of advanced lung cancer with metastatic bone lesions, for surveillingthe surveillance of molecular subtype switching of in metastatic breast cancer cells and identifying the identification of bone marrow involvement in newly diagnosed cases of lymphoma [2122]. However, no studies have examined the utility of PET/CT-guided targeted biopsy for prostate pathologies. In this study, therefore, we evaluated the safety and efficacy of PET/CT-fusion-guided percutaneous trans-pararectal space prostate biopsy for the diagnosis of prostate cancer.

\section{Methods}

\section{Patients}

A retrospective review of our electronic database comprising 14 patients (age range, 52-78 years; mean age, 69.0 years) identified who were retrospectively enrolled in our study. Among which, 13 patients had indications of prostate cancer based on an elevated PSA level, abnormalities detected during a digital rectal examination (DRE), abnormal imaging findings or a combination thereof. One patient underwent prior treatment for prostate cancer. All 14 patients underwent ${ }^{18} \mathrm{~F}$-fluorodeoxyglucose (FDG) PET/CT scans. The diagnoses of prostate cancer were confirmed via histopathological examination or evidence of prostate cancer detected during a clinical and imaging follow-up 12 months after the initial PET/CT examination.

\section{PET/CT and image interpretation}

The patients were asked to fast for at least 4 hours before undergoing ${ }^{18} \mathrm{~F}-\mathrm{FDG}$ PET/CT to ensure a normal blood glucose level $(70-120 \mathrm{mg} / \mathrm{dL})$ before the intravenous injection of ${ }^{18} \mathrm{~F}-\mathrm{FDG}$. Each patient received an intravenous injection of $370-666 \mathrm{MBq}(10-18 \mathrm{mCi})$ of ${ }^{18} \mathrm{~F}-\mathrm{FDG}$. Data were acquired using a PET/CT system (Discovery STE; GE Medical Systems, Milwaukee, WI, USA) at 45 min post-injection. The following procedure was used to acquire data First, a CT scan was performed from the head to the pelvic floor, using the following settings: $110 \mathrm{kV}, 110 \mathrm{~mA}$, tube rotation time of $0.5 \mathrm{~s}$, and 3.3-mm scan section thickness. These settings were matched to the PET settings. Immediately after the CT scan, a PET scan was performed to obtain a transverse field of view identical to that obtained by $\mathrm{CT}$. The acquisition time was 3 minutes per table position. 
Based on our previous knowledge of the normal biodistribution of ${ }^{18} \mathrm{~F}-\mathrm{FDG}$, the malignant prostate cancer lesions were identified as foci exhibiting increased tracer accumulation relative to the cancer-free contralateral structures and surrounding soft tissues. The lesions were graded qualitatively as definitely or probably abnormal (i.e., representative of a tumour) if a moderate to marked increase in the accumulation of ${ }^{18} \mathrm{~F}$-FDG was observed. A diffused, mild increase or no increase in tracer accumulation (i.e., abnormalities identified by CT for which no corresponding abnormality was visualised by PET) was indicative of pathologically normal or benign tissue.

\section{PET/CT-fusion-guided percutaneous trans-pararectal space prostate biopsy}

On the day after the whole-body PET/CT scan, a board-certified interventional radiologist and nuclear medicine physician performed the prostate biopsies in a PET/CT suite dedicated to biopsy procedures. PET/CT-guided prostate biopsies were performed in a stepwise manner under aseptic conditions, with the patient in a prone position. A local anaesthetic agent, lidocaine, was administered subcutaneously via the buttocks. An 18-gauge biopsy needle (Magum, Bard, AZ, USA) was introduced in a stepwise manner under the guidance of PET/CT fusion and CT imaging via a percutaneous trans-pararectal space approach, without damaging the rectal wall or skin of the perineum. Two prostate tissue specimens was obtained from each patient and were subjected to subsequent histopathological and immunohistochemical examinations. The PET/CT-fusion-guided percutaneous trans-pararectal space prostate biopsy approach is shown in detail in Figure 1. Representative ${ }^{18} \mathrm{~F}-\mathrm{FDG}$ PET/CT images of patients undergoing prostate needle biopsy are shown in Figures 2 and 3.

\section{Synchronised multiple organ biopsy}

Of the 14 patients in the electronic database, synchronous pulmonary lesions were detected in 3 patients, hypermetabolic bone lesions were observed in 3 patients, a retroperitoneal mass was detected in 1 case, and an renal mass was found in 1 patient incidentally. These 8 patients underwent PET/CT-guided percutaneous biopsies targeting the ${ }^{18} \mathrm{~F}$-FDG-avid regions.

\section{Clinical and imaging follow-up of the prostate lesions}

All biopsy results were confirmed histologically or clinically, and a final diagnosis was made for all patients. For prostatic lesions which were pathologically not indicative of malignancy, clinical and imaging follow-ups were performed for at least 12 months after the PET/CT examination. 
Statistical analysis

The diagnostic accuracy rate of PET/CT and the diagnostic success rate of prostate biopsy were determined based on the number of confirmed cases, rather than the number of lesions.

\section{Results}

Diagnostic accuracy of ${ }^{18}$ F-FDG PET/CT for identifying malignant prostatic lesions

The diagnostic accuracy of ${ }^{18} \mathrm{~F}-\mathrm{FDG}$ PET/CT was determined by histopathological examination or based on other clinical evidence. The ${ }^{18} \mathrm{~F}$-FDG PET/CT scans of 14 patients of prostatic lesion led to the identification of $9,0,3$, and 2 cases with true positive, false negative, true negative, and false positive results, respectively. The latter 2 cases were subsequently identified as chronic prostatitis and granulomatous prostatitis. The diagnostic accuracy of ${ }^{18} \mathrm{~F}-\mathrm{FDG}$ PET/CT for the detection of prostate cancer was $81.8 \%(9 / 11$; Table 2$)$.

Diagnostic accuracy of PET/CT-guided prostate biopsy for identifying malignant prostatic lesions

The diagnostic accuracy of PET/CT-guided prostate biopsy for prostate cancer was determined according to a histopathological examination or further clinical evidence. The histopathological analysis of PET/CTguided prostate biopsy specimens confirmed the diagnosis of prostate cancer in 9 cases and of benign lesions in 5 cases.

Of the 5 patients with benign lesions, 1 patient had undergone multiple prostate biopsies at our hospital because of an elevated PSA level since 2013 but received biopsy results that were negative for prostate cancer. Each time, the patient's PSA level decreased after symptomatic treatment, and eventually a negative result was achieved after 6 years of follow-up. Another patient was infected with human immunodeficiency virus. Because the histopathological analysis of the prostate biopsy yielded negative results, special staining and molecular detection techniques were applied. Acid-fast staining of the specimen revealed bacteria, and a polymerase chain reaction assay indicated the presence of tuberculosis-associated genes. The prostatic lesion was attributed to mycobacterial infection. Although ${ }^{18} \mathrm{~F}$-FDG PET/CT incidentally detected hypermetabolic nodules in the prostate of the $6^{\text {th }}$ and $7^{\text {th }}$ patients, the serum PSA level and DRE evaluations did not suggest malignancy, and the results were similar to those detected in the second year of follow-up. The $8^{\text {th }}$ patient underwent PET/CT-guided biopsy because of an increased PSA level, but the results were negative for prostate cancer. Similar results were obtained with subsequent TRUS-guided prostate biopsy. The patients were followed for 1 year after biopsy. The diagnostic success rate of ${ }^{18} \mathrm{~F}$-FDG PET/CT-guided percutaneous trans-pararectal space prostate biopsy was $100.0 \%(14 / 14$; Table 3$)$. 
Simultaneous PET/CT-guided biopsies of the prostate and other organs were performed in $57.1 \%(8 / 14)$ of the patients with prostatic lesions. The incidence rate of multiple primary tumours was $28.6 \%(4 / 14)$. The patients' characteristics are summarised in Table 1.

\section{Biopsies of primary prostate and bone lesion}

Simultaneous PET/CT-guided prostate and bone lesion biopsies were performed in $28.6 \%(4 / 14)$ of the patients. Histopathological examinations confirmed the presence of metastatic bone lesions in patients with prostate cancer (Table 1).

\section{Complications of targeted prostate biopsy}

Each targeted prostate biopsy required an average of approximately 20 minutes. Slight bleeding and pain were experienced by almost all patients who underwent the biopsy. However, no serious complications were noted. Moreover, no complications associated with rectal puncture injury occurred in the patients who underwent prostate biopsy (Table 4).

\section{Discussion}

According to the 2015 Annual Report of the National Central Cancer Registry (China), the overall incidence of prostate cancer was 7.1/10 $0^{5}$ individuals in 2011. Accordingly, prostate cancer was the ninth most prevalent cancer overall, and seventh most prevalent among men [23]. However, the majority of patients with prostate cancer were diagnosed at an advanced disease stage. Early diagnosis is a key driver of improved survival among men with prostate cancer in China. Organ-confined prostate cancer at the time of diagnosis is associated with a good prognosis and a favourable response to curative therapy [24]. Conversely, metastatic prostate cancer is associated with a poor prognosis, and skeletal metastasis is associated with a 5 -year survival rate of $<30 \%$ [25].

In the present study, 13 patients presented with indications of prostate cancer, including an elevated PSA level and/or abnormal digital rectal examination and/or imaging findings. One patient had been treated previously for prostate cancer. According to the ${ }^{18} \mathrm{~F}$-FDG PET/CT findings, 6 patients $(66.7 \%)$ with prostate cancer had skeletal metastases, and 1 patient (11.1\%) presented with a tumour that was no longer organ-confined and had invaded the bladder. The incidence rate of multiple primary tumours among patients with prostate cancer was $28.6 \%(4 / 14)$.

Improvements in cancer care have been linked closely to advances in imaging technologies, as these improvements enable a more accurate diagnosis, staging, and surveillance of disease. PET/CT recently 
emerged as a promising diagnostic imaging platform for both primary and recurrent prostate cancers [26]. In addition to ${ }^{18} \mathrm{~F}-\mathrm{FDG}$, routine clinical imaging procedures use various radiolabelled tracers [e.g., ${ }^{18} \mathrm{~F}$ choline, ${ }^{18} \mathrm{~F}-\mathrm{NaF},{ }^{68} \mathrm{Ga}$-prostate-specific membrane antigen (PSMA), ${ }^{68} \mathrm{Ga}$-DOTATATE, ${ }^{18} \mathrm{~F}$-FACBC] with demonstrated efficacy for cancer diagnosis in various clinical settings [26-27]. Newly developed tracers exhibit increased accuracy for the detection of small, incipient metastatic foci [28-29]. Improvements in MRI techniques, and particularly in functional imaging, have enabled radiologists to play an important role in the risk stratification and management of patients [30-32].

${ }^{18} \mathrm{~F}$-FDG PET/CT is primarily useful in the diagnosis and staging of prostate cancer because it combines the metabolic information of PET with the anatomic information of CT. Moreover, whole-body scanning facilitates the detection of primary tumours in multiple organs. Additionally, ${ }^{18} \mathrm{~F}-\mathrm{FDG}$ PET/CT can accurately diagnose cancer recurrence. In this study, prostate cancer was confirmed via histopathological examinations in 9 of 14 patients, whereas the remaining 5 patients had benign lesions (including 2 misdiagnoses of prostate cancer). The diagnostic accuracy of ${ }^{18} \mathrm{~F}-\mathrm{FDG}$ PET/CT for prostate cancer was $81.8 \%(9 / 11)$. Of the 14 patients, we detected multiple primary tumours that were confirmed pathologically as prostate cancer in 4 patients (28.6\%). One of the 9 patients exhibited a tumour recurrence and metastatic bone lesion on ${ }^{18} \mathrm{~F}-\mathrm{FDG} \mathrm{PET} / \mathrm{CT}$, which was eventually confirmed as prostate cancer.

Despite the enthusiasm surrounding the use of PET and MRI for diagnosing prostate cancer, prostate biopsy remains the gold-standard diagnostic option. Prostate biopsies have been used to diagnose prostate cancer since the beginning of the last century [5-6]. Ideally, prostate biopsy should be minimally invasive with few side effects, and should identify a large proportion of men who would benefit from treatment while minimising the identification of men with clinically insignificant cancer to prevent overtreatment. Although the optimal method of prostate biopsy remains controversial, TRUS-guided biopsy is the most widely accepted method for the diagnosis of prostate cancer. Greyscale TRUS has a low sensitivity and specificity for prostate cancer detection, and some studies reported low detection rates for saturation prostate biopsy via the trans-rectal or trans-perineal route (21.7-45\% [33-36] and 22.7-42.2\%, respectively [37-39]). Despite the advantages of TRUS-guided prostate biopsy, its invasive nature and rectal approach could potentially cause complications. Infectious complications ranging from asymptomatic bacteriuria to septic shock may occur during TRUS-guided prostate biopsy via the manipulation of infected prostate tissue, which also increases the risk of introducing the rectal flora into the prostate tissue, urine, and blood.

Here, we introduce a novel approach to prostate biopsy, namely ${ }^{18}$ F-FDG PET/CT-guided percutaneous trans-pararectal space prostate biopsy. PET/CT is a widely accepted modality for detecting prostatic lesions and identifying cancer recurrence and metastasis, particularly when conventional imaging findings are equivocal or conflicting. PET/CT also increases the choice of biopsy location, which can increase the associated accuracy and safety. 
Besides the primary lesion, PET/CT also enables the biopsy of other metabolically active lesions, such as metastatic bone lesions that cannot be detected using conventional imaging methods. In such cases, PET/CT-guided biopsy allows the sampling of multiple hypermetabolic lesions, thereby reducing falsenegative or false-positive biopsy rates. In our study, PET/CT-guided biopsies were performed in 14 patients with indications of prostate cancer based on PET/CT images. The $4^{\text {th }}$ patient in our study underwent prostate, lung, and iliac bone biopsy under PET/CT guidance, despite the lack of apparent bone morphological changes on a CT scan. The histopathological analysis revealed that both the prostatic and pulmonary lesions had originated from the primary prostate tumour, whereas the bone lesions had metastasised from the lung lesion rather than the prostate tumour.

Before performing PET/CT-guided percutaneous ${ }^{18} \mathrm{~F}$-FDG-avid target biopsy, we selected the optimal puncture site based on the target location to ensure a favourable biopsy success rate and the optimal needle path to minimise trauma. When selecting the target location, the site with the highest metabolic ${ }^{18} \mathrm{~F}-\mathrm{FDG}$ accumulation among all focal hypermetabolic prostate lesions was selected first, as such lesions would more likely represent the true cancer grade and would thus affect the clinical classification, staging, and prognosis. Other visible but controversial lesions were targeted thereafter.

The optimal needle path is extremely important when aiming to avoid puncturing the nearby blood vessels, spinal nerve trunk, and other vital organs. In our study, we chose the anatomically safer transpararectal space, rather than the trans-rectal or trans-perineal approach. This option can avoid the damage to the rectum or perineum associated with a conventional biopsy path, and may minimise the occurrence of complications. Altogether, we considered these factors to ensure a safe, feasible, and effective prostate biopsy procedure. ${ }^{18} \mathrm{~F}$-FDG PET/CT-guided percutaneous trans-pararectal space prostate biopsy is the third prostate biopsy technique worldwide that can accommodate all these factors simultaneously. This novel method can reduce the false-negative or false-positive rate and is a relatively safer and more effective way to obtain a pathological diagnosis.

In our study, a correct biopsy-assisted diagnosis was made on all cases. The diagnostic success of ${ }^{18} \mathrm{~F}-$ FDG PET/CT-guided percutaneous trans-pararectal space prostate biopsy was $100.0 \%$ (14/14).

Simultaneous PET/CT-guided targeted biopsies of the prostate and other organs were performed in $57.1 \%$ $(8 / 14)$ of the patients. Finally, histopathological examination confirmed the presence of primary prostate lesions in 4 patients. In summary, ${ }^{18}$ F-FDG PET/CT-guided percutaneous trans-pararectal space prostate biopsy mitigates the issue of trans-visceral puncture trauma by accurate biopsy, and enables a pathological diagnosis from a smaller tissue volume. Moreover, the ability to biopsy other organs simultaneously provides additional diagnostic possibilities, thus avoiding an unnecessary diagnosis or over-treatment.

Our study had several limitations. First, the small sample size might have limited the statistical significance of the results. Second, ${ }^{18}$ F-FDG PET/CT is associated with some disadvantages. For example, small tumours might remain undetected because of partial-volume effects, which would cause falsely low measurements of the true ${ }^{18} \mathrm{~F}-\mathrm{FDG}$ activity level. Moreover, ${ }^{18} \mathrm{~F}-\mathrm{FDG}$ frequently accumulates in 
areas of inflammation. However, physiological variants and benign pathological causes of ${ }^{18} \mathrm{~F}$-FDG uptake can be recognised specifically and categorised properly using other approaches. Accordingly, we will evaluate the efficacy of ${ }^{68} \mathrm{Ga}$-PSMA PET/CT-guided prostate biopsy in a future study.

\section{Conclusion}

Conventional prostate biopsy methods remain associated with several risks and side effects. In this study, we present a new molecular imaging-guided approach to the targeted biopsy of prostate lesions. PET/CT-fusion-guided percutaneous trans-pararectal space prostate biopsy may provide an effective approach to the diagnosis of prostate cancer, and can be performed safely without any complications associated with trans-rectal puncture.

\section{List Of Abbreviations}

CT, computed tomography; FDG, fluorodeoxyglucose; PET, positron emission tomography; PSA, prostatespecific antigen; PSMA, prostate-specific membrane antigen; TRUS, trans-rectal ultrasonography; MIP, maximum intensity projection

\section{Declarations}

\section{Ethics approval and consent to participate}

All procedures performed in studies involving human participants were in accordance with the ethical standards of the institutional and/or national research committee and with the principles of the1964 Declaration of Helsinki and its later amendments or comparable ethical standards. However, this was a retrospective analysis and for this type of study formal consent is not required. Informed consent was obtained from all individual participants included in the study. All patients gave their written informed consent prior to intervention.

\section{Consent for publication}

The healthcare professionals depicted in the lower right of Figure 1 provided written informed consent for publciation of this photograph.

\section{Availability of data and materials}

The datasets and materials used and/or analyzed during the current study are available from the corresponding author on reasonable request.

\section{Competing interests}

The authors declare that they have no competing interests 


\section{Funding}

This study was funded by the National Natural Science Foundation of China (81101067), the The key medical and health projects in Xiamen (3502Z20191104). Funding bodies were not involved in the design of the study, collection, analysis, and interpretation of date or in writing the manuscript. Funding bodies were not involved in the design of the study, collection, analysis, and interpretation of date or in writing the manuscript.

\section{Authors' contributions}

NL, LS carried out conception and design. NL, DR, YP, HC, WG, BH carried out collection and assembly of data. NL, DR, QS and BH carried out data analysis and interpretation. BH, DR and YP carried out PET/CT acquisition and collection of clinical data. HW, LS and HC provided critical comments for this manuscript. NL, DR, YP, HW and LS participated in manuscript writing and revision. All authors read and approved the final manuscript.

\section{Acknowledgements}

We are indebted to Nuclear department \& Minnan PET Center of Fujian Province for providing technical assistance. We also thank all the patients for allowing us to analyze their data.

\section{References}

1. Bray F, Ferlay J, Soerjomataram I, Siegel RL, Torre LA, Jemal A. Global Cancer Statistics 2018: GLOBOCAN Estimates of Incidence and Mortality Worldwide for 36 Cancers in 185 Countries. CA Cancer J Clin. 2018;68:394-424.

2. Gu XY, Zheng RS, Zhang SW, Zeng HM, Sun KX, Zou XN, Xia CF, Yang ZX, Li H, Chen WQ, He J. Analysis on the trend of prostate cancer incidence and age change in cancer registration areas of China, 2000 to 2014. Zhonghua Yu Fang Yi Xue Za Zhi.2018;52:586-92.

3. Han SJ, Zhang SW, Chen WQ, Li CL. Analysis of the status and trends of prostate cancer incidence in China. Chinese Clinical Oncology. 2013;18:330-4.

4. Mohammed W, Davis NF, Elamin S, Ahern P, Brady CM, Sweeney P. Six-core versus twelve-core prostate biopsy: a retrospective study comparing accuracy, oncological outcomes and safety. Ir J Med Sci. 2016;185:219-23.

5. Torp-Pedersen S, Lee F, Littrup PJ, Siders DB, Kumasaka GH, Solomon MH, McLeary RD. Transrectal biopsy of the prostate guided with transrectal US: Longitudinal and multiplanar scanning. Radiology. 1989;170:23-7.

6. Hodge KK, McNeal JE, Terris MK, Stamey TA. Random systematic versus directed ultrasound guided transrectal core biopsies of the prostate. J Urol. 1989;142:71-4.

7. Shim HB, Park HK, Lee SE, Ku JH. Optimal site and number of biopsy cores according to prostate volume prostate cancer detection in Korea. Urology. 2007;69:902-6.8. 
8. Elabbady AA, Khedr MM. Extended 12-core prostate biopsy increases both the detection of prostate cancer and the accuracy of Gleason score. Eur Urol. 2006;49:49-53.

9. Gore JL, Shariat SF, Miles BJ, Kadmon D, Jiang N, Wheeler TM, Slawin KM. Optimal combinations of systematic sextant and laterally directed biopsies for the detection of prostate cancer. J Urol. 2001;165:1554-9.

10. Philip J, Ragavan N, Desouza J, Foster CS, Javle P. Effect of peripheral biopsies in maximising early prostate cancer detection in 8-, 10- or 12-core biopsy regimens. BJU Int. 2004;93:1218-20.

11. Scattoni V, Roscigno M, Raber M, Deho F, Maga T, Zanoni M, Riva M, Sangalli M, Nava L, Mazzaccoli B, Freschi M. Initial extended transrectal prostate biopsy-are more prostate cancers detected with 18 cores than with 12 cores?. J Urol. 2008;179:1327-31.

12. Pepe P, Aragona F. Saturation prostate needle biopsy and prostate cancer detection at initial and repeat evaluation. Urology. 2007;70:1131-5.

13. Jones JS, Patel A, Schoenfield L, Rabets JC, Zippe CD, Magi-Galluzzi C. Saturation technique does not improve cancer detection as an initial prostate biopsy strategy. J Urol. 2006;175:485-8.

14. Schoots IG, Roobol MJ, Nieboer D, Bangma CH, Steyerberg EW, Hunink MM. Magnetic Resonance Imaging-targeted Biopsy May Enhance the Diagnostic Accuracy of Significant Prostate Cancer Detection Compared to Standard Transrectal Ultrasound-guided Biopsy: A Systematic Review and Meta-analysis. Eur Urol. 2015;68:438-50.

15. Pepe P, Garufi A, Priolo G, Pennisi M. Transperineal Versus Transrectal MRI/TRUS Fusion Targeted Biopsy: Detection Rate of Clinically Significant Prostate Cancer. Clin Genitourin Cancer. 2016;15:336.

16. Kasivisvanathan V, Rannikko AS, Borghi M, Panebianco V, Mynderse LA, Vaarala MH, Briganti A, Budaus L, Hellawell G, Hindley RG, Roobol MJ. MRI-Targeted or Standard Biopsy for Prostate-Cancer Diagnosis. N Engl J Med. 2018;378:1767-77.

17. Farag M, Riddell S, Daffy J, Wong LM. Comparing infective complications from transrectal ultrasound guided prostate biopsy following transition to single dose oral ciprofloxacin prophylaxis. Investig Clin Urol. 2019;60:54-60.

18. Lee MS, BS, Cho JY, Kim SY, Cheon GJ, Moon MH, Oh S, Lee J, Lee S, Woo S, Kim SH. Diagnostic Value of Integrated PET/MRI for Detection and Localization of Prostate Cancer: Comparative Study of Multiparametric MRI and PET/CT. J Magn Reson Imaging. 2017;45:597-609.

19. Schuster DM, Nanni C. PET Tracers Beyond FDG in Prostate Cancer. Semin Nucl Med. 2016;46:50721.

20. Jadvar H. Prostate Cancer: PET with ${ }^{18} \mathrm{~F}$-FDG, ${ }^{18} \mathrm{~F}$ - or ${ }^{11} \mathrm{C}$-Acetate,and ${ }^{18} \mathrm{~F}$ - or ${ }^{11} \mathrm{C}$-Choline. J Nucl Med. 2011;52:81-9.

21. Guo W, Hao B, Luo N, Ruan D, Guo X, Chen HJ, Wu H, Sun L. Early re-staging and molecular subtype shift surveillance of locally recurrent or metastatic breast cancer: A new PET/CT integrated precise algorithm. Cancer Lett. 2018;418:221-29. 
22. Hao B, Zhao L, Luo NN, Ruan D, Pang YZ, Guo W, Fu H, Guo XY, Luo ZM, Wu J, Chen HJ. Is it sufficient to evaluate bone marrow involvement in newly diagnosed lymphomas using ${ }^{18}$ F-FDG $\mathrm{PET} / \mathrm{CT}$ and/or routine iliac crest biopsy? A new approach of PET/CT-guided targeted bone marrow biopsy, BMC Cancer. 2018;18:1192.

23. Chen W, Zheng R, Zeng H, Zhang S, He J. Annual report on status of cancer in China, 2011. Chin J Cancer Res. 2015;27:2-12.

24. Peyromaure M, Debré B, Mao K, Zhang G, Wang Y, Sun Z, Xu D, Jiang J, Sun $Y$. Management of prostate cancer in China: a multicenter report of 6 institutions. $J$ Urol.2005;174:1794-7.

25. KW Wong, WK Ma, CW Wong, MH Wong, CF Tsang, HL Tsu, KL Ho, MK Yiu. Impact of skeletal-related events on survival in patients with metastatic prostate cancer prescribed androgen deprivation therapy. Hong Kong Med J. 2016;22:106-15.

26. Li R, Ravizzini GC, Gorin MA, Maurer T, Eiber M, Cooperberg MR, Alemozzaffar M, Tollefson MK, Delacroix SE, Chapin BF. The use of PET/CT in prostate cancer. Prostate Cancer Prostatic Dis. 2018;21:4-21.

27. Ceci F, Herrmann K, Hadaschik B, Castellucci P, Fanti S. Therapy assessment in prostate cancer using choline and PSMA PET/CT. Eur J Nucl Med Mol Imaging.2017;44:78-83.

28. Nitsch S, Hakenberg OW, Heuschkel M, Dräger D, Hildebrandt G, Krause BJ, Schwarzenbock SM. Evaluation of Prostate Cancer with ${ }^{11} \mathrm{C}$ - and ${ }^{18}$ F-Choline PET/CT: Diagnosis and Initial Staging. J Nucl Med. 2016;57:38-42.

29. Zang S, Shao G. ${ }^{68} \mathrm{Ga}-\mathrm{PSMA}-11 \mathrm{PET} / \mathrm{CT}$ for prostate cancer staging and risk stratification in Chinese patients. Oncotarget. 2017;8:12247-58.

30. Koo PJ, Kwak JJ, Pokharel S, Choyke PL. Novel Imaging of Prostate Cancer with MRI, MRI/US, and PET. Curr Oncol Rep. 2015;17:56-71.

31. Lindenberg L, Ahlman M, Turkbey B, Mena E, Choyke PL. Evaluation of Prostate Cancer with PET/MRI. J Nucl Med. 2016;57:111-6.

32. Lee MS, Cho JY. Diagnostic Value of Integrated PET/MRI for Detection and Localization of Prostate Cancer: Comparative Study of Multiparametric MRI and PET/CT. J Magn Reson Imaging. 2017;45:597-609.

33. de la Taille A, Antiphon P, Salomon L, Cherfan M, Porcher R, Hoznek A, Saint F, Vordos D, Cicco A, Yiou R, Zafrani ES. Prospective evaluation of a 21-sample needle biopsy procedure designed to improve the prostate cancer detection rate. Urology. 2003;61:1181-6.

34. Patel AR, Jones JS, Rabets J, DeOreo G, Zippe CD. Parasagittal biopsies add minimal information in repeat saturation prostate biopsy. Urology. 2004;63:87-9.

35. Simon J, Kuefer R, Bartsch G Jr, Volkmer BG, Hautmann RE, Gottfried HW. Intensifying the saturation biopsy technique for detecting prostate cancer after previous negative biopsies: a step in the wrong direction. BJU Int. 2009;102:459-62. 
36. Walz J, Graefen M, Chun FK, Erbersdobler A, Haese A, Steuber T, Schlomm T, Huland H, Karakiewicz $\mathrm{PI}$. High incidence of prostate cancer detected by saturation biopsy after previous negative biopsy series. Eur Urol. 2006;50:498-505.

37. Satoh T, Matsumoto K, Fujita T et al. Cancer core distribution in patients diagnosed by extended transperineal prostate biopsy. Urology. 2005;66:114-8.

38. Merrick GS, Gutman S, Andreini H, Taubenslag W, Lindert DL, Curtis R, Adamovich E, Anderson R, Allen Z, Butler W, Wallner K. Prostate cancer distribution in patients diagnosed by transperineal template-guided saturation biopsy. Eur Urol. 2007;52:715-23.

39. Bott SR, Henderson A, Halls JE, Montgomery BS, Laing R, Langley SE. Extensive transperineal template biopsies of prostate: modified technique and results. Urology. 2006;68:1037-41.

\section{Tables}

Due to technical limitations the Tables are available as a download in the Supplementary Files.

\section{Figures}
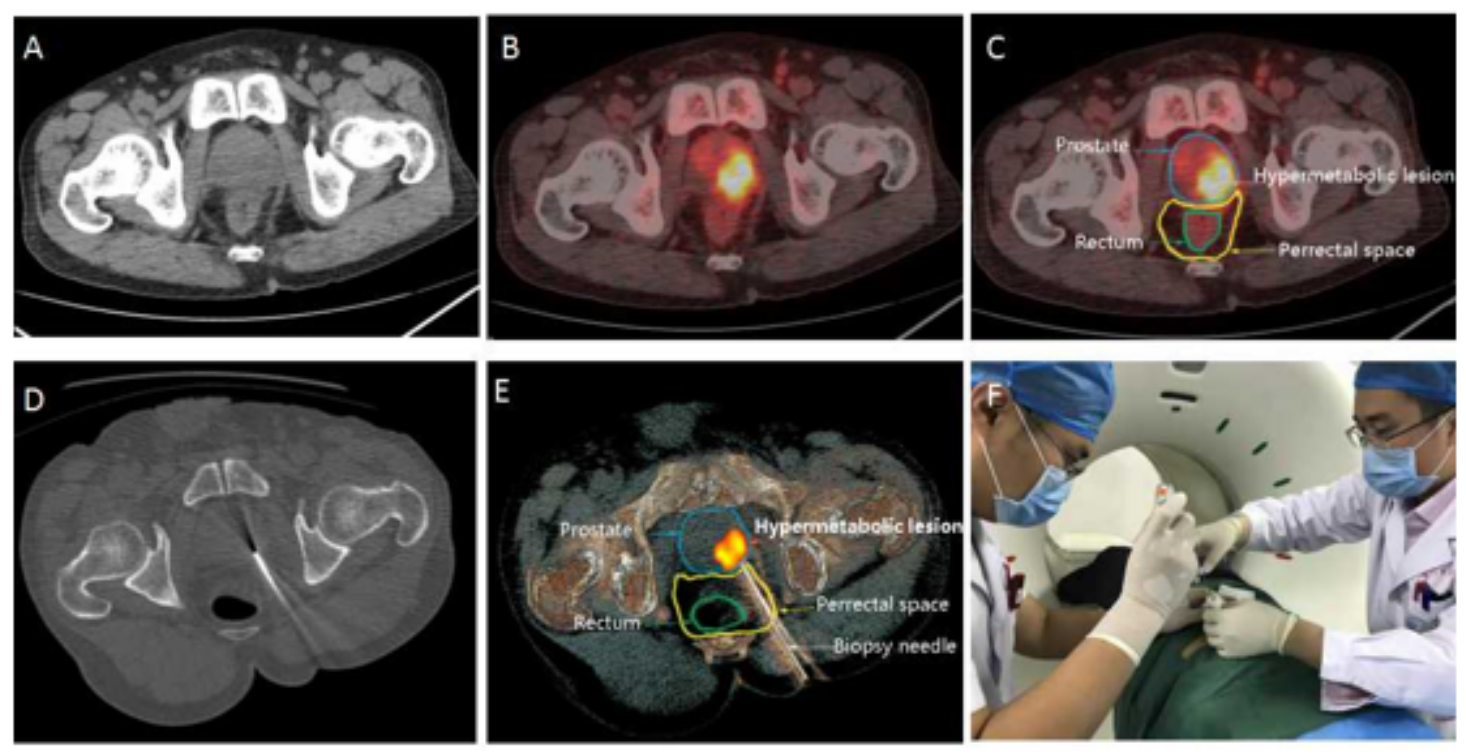

\section{Figure 1}

Workflow of the PET/CT-fusion-guided percutaneous trans-pararectal space prostate biopsy approach. Axial CT (A) revealed no obvious abnormality. Fused PET/CT (B) incidentally detected an isolated hypermetabolic lesion in the left prostatic lobe. The outline of the anatomical structure on PET is shown (C). The patient underwent PET/CT-guided percutaneous trans-pararectal space targeted biopsy of the left lobe nodule $(D, E)$. The targeted biopsy process $(F)$. 

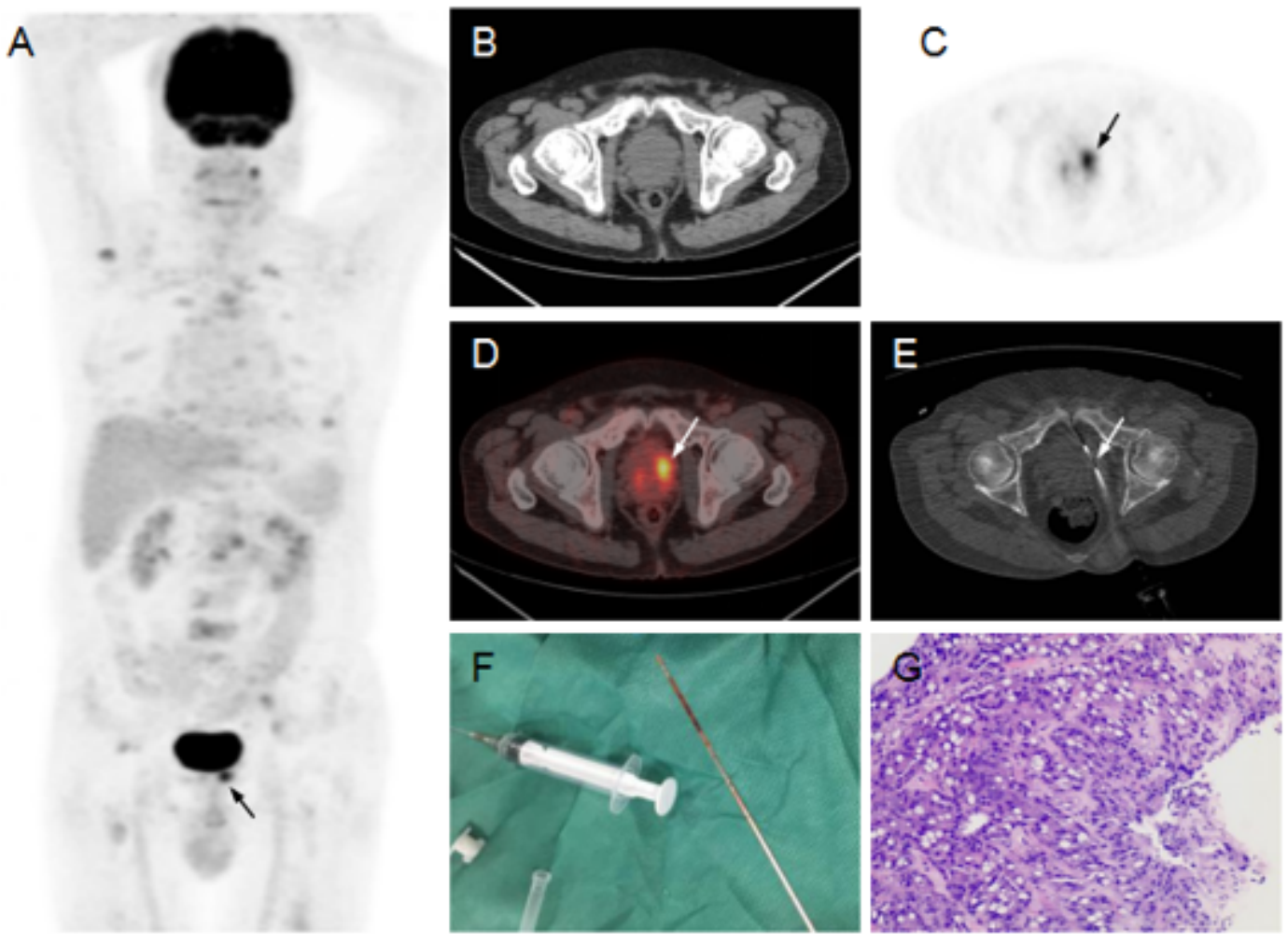

\section{Figure 2}

The patient was hospitalised with fever, a 2-month history of subcutaneous nodules, and an elevated serum prostate specific antigen (PSA) level $(13.28 \mathrm{ng} / \mathrm{ml}$; reference range, $0-4 \mathrm{ng} / \mathrm{ml})$. To determine the aetiology of fever and increased PSA, the patient underwent whole-body $18 \mathrm{~F}$ - fluorodeoxyglucose (FDG) PET/CT. A PET MIP image (A) revealed diffusely increased 18F-FDG uptake in the systemic bone and a focal area of high metabolism within the prostatic region. Fused PET/CT (B) and PET (C) detected an incidentally isolated hypermetabolic lesion with a diameter of $1.1 \mathrm{~cm}$ in the left prostate lobe (maximum standard uptake value (SUV), 5.18; average SUV, 2.67). Axial CT (D) did not reveal obviously abnormalities. The patient underwent 18F-FDG PET/CT-guided percutaneous trans-pararectal space targeted biopsy (E-F) of the left lobe nodule. A postoperative pathologic analysis of the tissue specimen $(G)$ was highly indicative of adenocarcinoma (Gleason score $3+4=7)(J)$. The bone lesions $(H)$ were subjected to 18F-FDG PET/CT-guided bone biopsy (I), and a pathological analysis revealed that hematopoietic activation of bone marrow was drug-induced. 


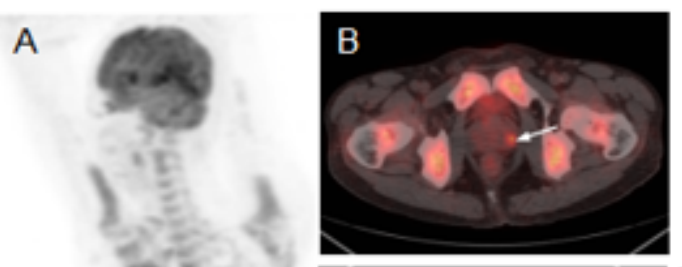

C
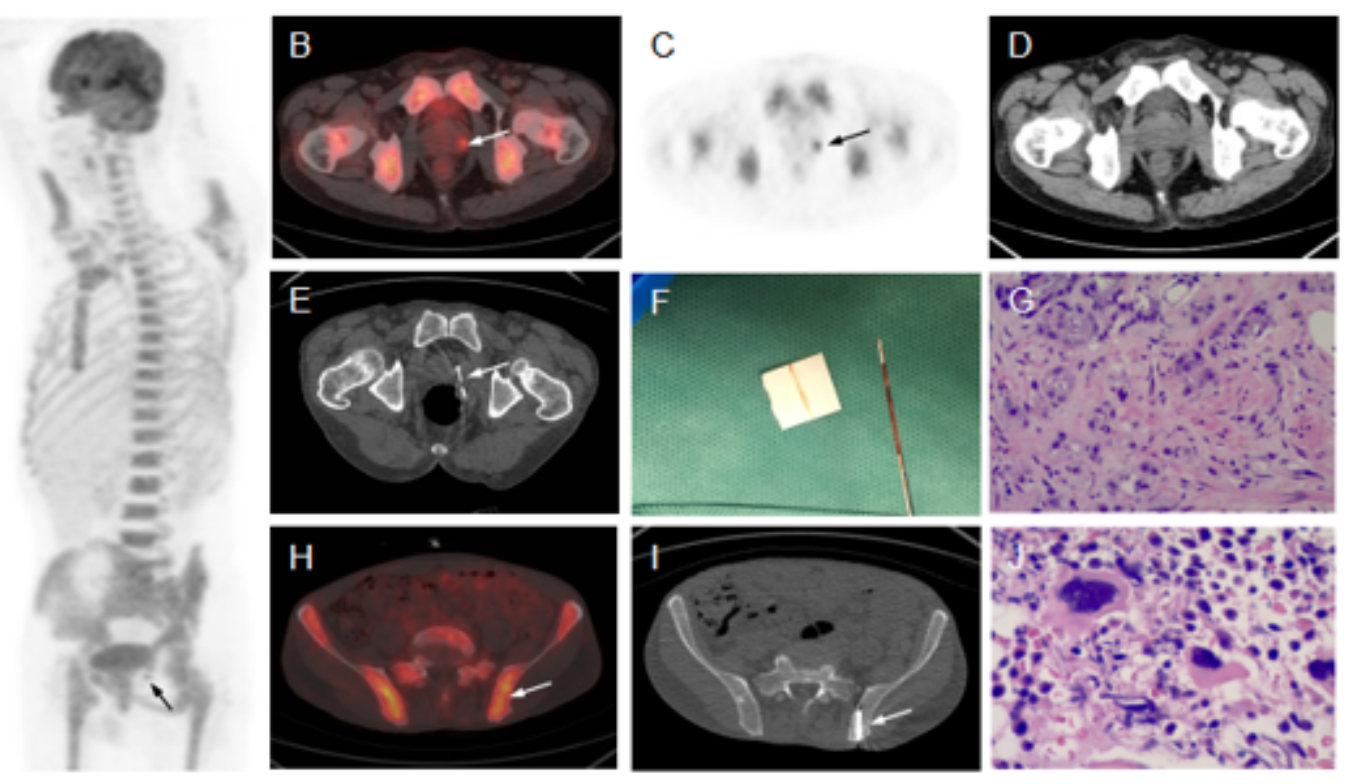

\section{Figure 3}

The patient presented with a 1-month history of back pain and weakness of both lower extremities for half a month. A previous MRI scan of the lumbar region revealed a suspected thoracic tumour. After admission, the patient had an elevated serum prostate specific antigen (PSA) level $(1513 \mathrm{ng} / \mathrm{ml}$; reference range, $0-4 \mathrm{ng} / \mathrm{ml}$ ). He was referred to our centre for whole-body $18 \mathrm{~F}$-fluorodeoxyglucose (FDG) PET/CT, which revealed strong focal uptake within the left prostate lobe (maximum standard uptake value (SUV), 9.30; average SUV, 7.46) (PET MIP image, A). Although axial CT (B) yielded negative results, PET (C) and fused PET/CT (D) indicated an isolated highly metabolic nodule with a diameter of $2.0 \mathrm{~cm}$. Axial CT (EF) distinctly and intuitively enabled PET/CT-guided targeted prostate biopsy. The corresponding histopathological analysis of the puncture specimen revealed prostate adenocarcinoma (Gleason score $4+5=9)(G)$.

\section{Supplementary Files}

This is a list of supplementary files associated with this preprint. Click to download.

- Tables.pdf 